\title{
Recuperação por desbridamento manual da valva aórtica estenótica calcificada
}

Renato A. K. KALIL*, Guaracy F. TEIXEIRA FILHO*, João Ricardo M. SANT' ANNA*, Paulo R. PRATES $^{\star}$, Fernando A. LUCCHESE ${ }^{\star}$, Carla R. BRAUCH ${ }^{\star}$, Edemar M. C. PEREIRA ${ }^{\star}$, Altamiro R. da COSTA $^{*}$, Marisa F. SANTOS ${ }^{*}$, Ivo A. NESRALLA*

RBCCV 44205-260

KALIL, R. A. K.; TEIXEIRA FILHO, G. F.; SANT'ANNA, J.R.M.; PRATES, P.R.; LUCCHESE, F. A.; BRAUCH, C. R.; PEREIRA, E. M. C.; COSTA, A. R.; SANTOS, M. F.; NESRALLA, I. A. - Recuperação por desbridamento manual da valva aórtica estenótica calcificada. Rev. Bras. Cir. Cardiovasc., 10 (2): 65-69, 1995.

RESUMO: Os autores analisam os resultados clínicos e evolução ecocardiográfica em uma série de pacientes em que foi possível recuperar a valva aórtica calcificada. Entre janeiro de 1993 e outubro de 1994, 31 pacientes foram considerados para recuperaçāo valvar, sendo obtido sucesso imediato em 21. A etiologia era congênita em 8 e senil em 13 pacientes; o sexo masculino em 10 e feminino em 11 e as idades variaram de 44 a 78 anos (média \pm DP $=63,8 \pm 9,5$ anos). Procedimentos associados foram 6 revascularizaçōes miocárdicas e 1 comissurotomia mitral, com tempos de perfusão de $53,8 \pm 21,4$ min e de isquemia miocárdica de $33,7 \pm 12,1 \mathrm{~min}$. O cálcio retirado com auxílio de pinças saca-cálcio usuais auxiliadas por aspiração e raspagem das válvulas. Em duas ocasiōes as válvulas foram perfuradas e suturadas com Propilene 5-0. Comissurotomia com bisturi foi realizada, se necessário. Dezesseis pacientes foram avaliados por ecocardiograma no pós-operatório imediato ou após $6 \mathrm{~m}$ a 1 ano. Todos os casos estāo em acompanhamento. Não houve mortalidade na fase hospitalar e 4,8\% (1 caso) na tardia. Não ocorreram complicações pós-operatórias significativas no período estudado, encontrando-se os pacientes em classe funcional I ou II. A avaliação ecocardiográfica mostrou gradiente instantâneo máximo na via de saída do VE de $90,7 \pm 23,3 \mathrm{mmHg}$ (média $\pm D P)$ no exame pré-operatório e $33,0 \pm 7,9$ no pós-operatório $(p<0,01)$. A espessura septal era $2,0 \pm 0,5 \mathrm{~cm}$ no pré e 1,2 $\pm 0,1$ no pós-operatório $(p<0,01)$. Insuficiência aórtica ao ecocardiograma pós-operatório foi considerada moderada em 2, leve em 9 e mínima em 5 casos. A recuperação da valva aórtica em estenose calcificada de etiologia congênita ou senil é factivel com resultado funcional bom, a curto e médio prazo. Nas calcificações senís consegue-se recuperação em maior número, porém a reconstrução valvar também é obtida em muitas estenoses congênitas calcificadas. Mesmo em válvulas severamente comprometidas por calcificaçōes, a recuperação funcional sem substituição deve ser tentada.

DESCRITORES: Estenose aórtica, cirurgia, desbridamento. Valvas cardiacas, cirurgia.

\section{INTRODUÇÃO}

A descalcificação mecânica e ultra-sônica foi empregada no passado, para recuperação de valvas aórticas estenóticas $1,4,9,10,11$. A mecânica havia sido praticamente abandonada há muito tempo, em favor das próteses 5; entretanto, alguns autores têm mostrado resultados favoráveis em situações espe- ciais. O desbridamento ultra-sônico foi abandonado devido à evolução de muitos casos para insuficiência aórtica a médio prazo ${ }^{3}$. A recuperação manual poderia trazer resultados melhores, devido a mais adequada preservação da estrutura fibrosa das válvulas. Mais recentemente, alguns centros retomaram as tentativas de recuperação de valvas aórticas calcificadas, especialmente de etiologia

Trabalho realizado no Instituto de Cardiologia do Rio Grande do Sul. Fundaçăo Universitária de Cardiologia. Porto Alegre, RS, Brasil.

Apresentado ao $22^{2}$ Congresso Nacional de Cirurgia Cardiaca. Brasília, DF, 30 de março a $1^{2}$ de abril, 1995.

* Do Instituto de Cardiologia do Rio Grande do Sul. Fundação Universitária de Cardiologia.

Endereço para correspondência: Renato A. K. Kalil. Av. Princesa Isabel, 395. CEP 90620-001 Porto Alegre, RS, Brasil.

Tel. [051] 223.5400 - Fax: [051] 217.1358 
KALIL, R. A. K.; TEIXEIRA FILHO, G. F.; SANT'ANNA, J. R. M.; PRATES, P. R.; LUCCHESE, F. A.; BRAUCH, C. R.; PEREIRA, E. M. C.; COSTA, A. R.; SANTOS, M. F.; NESRALLA, I. A. - Recuperaçăo por desbridamento manual da valva aórtica estenótica calcificada. Rev. Bras. Cir. Cardiovasc., 10 (2): 65-69, 1995.

senil, com resultados favoráveis inicialmente descritos $2,9,11-13$. Estimulados por tais informações e por sucessos em primeiras tentativas de descalcificação, iniciamos protocolo de investigação cirúrgica, retomando técnicas de desbridamento manual para tratar estenoses aórticas, senil ou congênita, calcificadas.

Este trabalho tem por objetivo analisar os resultados clínicos e a evolução ecocardiográfica em uma série de pacientes em que foi possível recuperar a valva aórtica calcificada.

\section{CASUÍSTICA E MÉTODOS}

Entre janeiro de 1993 e outubro de 1994, 31 pacientes, portadores de estenose aórtica calcificada, foram considerados para recuperação valvar. Não houve seleção pela gravidade dos casos, sendo realizados procedimentos, inclusive, em pacientes em choque cardiogênico.

Em 21 pacientes foi obtido sucesso imediato; em 10 outros foi necessária substituição por prótese; nestes 10 pacientes que sofreram implante de próteses, a etiologia era congênita em 9 e senil em 1. Na série de 21 casos recuperados que formam a base deste trabalho, a etiologia era congênita em 8 e senil em 13 vezes, sendo o sexo masculino em 10 e feminino em 11 pacientes; as idades variaram entre 44 e 78 anos (média \pm DP $=63,8 \pm 9,5$ anos). Foram realizadas revascularizações miocárdicas em 6 pacientes e comissurotomia mitral em 1, como procedimentos associados e o tempo médio de perfusão foi de $53,8 \pm 21,4 \mathrm{~min}$ e de isquemia miocárdica de $33,7 \pm 12,1 \mathrm{~min}$.

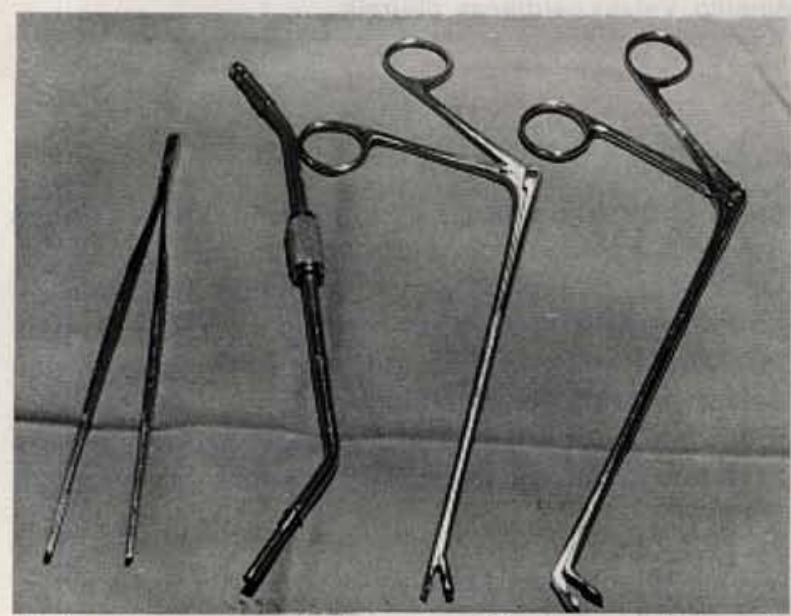

Fig. 1 - Instrumentos utilizados na descalcificaçāo por desbridamento manual: pinças saca-cálcio, aspirador com orifício terminale pinça anatômica.
Foi utilizada circulação extracorpórea empregando-se oxigenadores de bolhas ou de membrana, com hipotermia sistêmica moderada a $28^{\circ}$ a $30^{\circ} \mathrm{C}$ e parada isquêmica do coração sob cardioplegia hipotérmica cristalóide, drenando-se as cavidades esquerdas por aspirador em átrio esquerdo. A valva aórtica foi abordada por aortotomia oblíqua, antes de qualquer outro procedimento associado e as calcificacōes retiradas mediante pinças saca-cálcio usuais (Figura 1), auxiliadas por aspiração e raspagem das válvulas. Em 2 ocasiões, foram produzidas pequenas perfurações, suturadas com sucesso, utilizando-se pontos simples de Propilene 5-0. Cuidados foram tomados para não forçar a retirada de grandes fragmentos de tecido calcificado em blocos, preferindo-se a fragmentação por esmagamento com a pinça saca-cálcio ou por flexibilização forçada das válvulas, procedendo-se a raspagem e aspiração dos pequenos fragmentos com a ponta de aspirador metálico. Nas estenoses congênitas severamente calcificadas, iniciou-se pela retirada das calcificações maiores, seguida de comissurotomia, quando indicada. $\mathrm{Na}$ mobilização após a comissurotomia, conseguiu-se fragmentar as calcificações, de maneira a retirá-las com menor dificuldade. Independentemente do aspecto da valva à primeira vista, a recuperação foi tentada e muitas vezes obtida em situações em que o sucesso não se esperava, dada a primeira impressão (Figuras 2-A, B, C).

Todos os pacientes foram acompanhados nas fases hospitalar e tardia. Dezesseis foram avaliados por ecocardiograma no período pós-operatório imediato ou após 6 meses a 1 ano. O tempo de seguimento foi de 1 mês a 2 anos.

Os resultados foram expressos pela média mais ou menos desvio-padrão (m \pm DP) e a análise estatística realizada pelo teste $T$ de Student-Fisher para amostras pareadas, considerando-se o alfacrítico em $5 \%$, mas expressando os valores mais significativos, quando encontrados.

\section{RESULTADOS}

Não houve óbitos na fase hospitalar. Todos os pacientes receberam alta após 7 a 10 dias, exceto 1 paciente operado em choque cardiogênico, que necessitou assistência circulatória mecânica, com permanência hospitalar de 2 meses. No acompanhamento tardio ocorreu 1 óbito após 1 mês, devido a hemorragia digestiva, anemia, icterícia póstransfusional e provável sepsis. Não ocorreram outras complicações significativas no período estudado. Os pacientes encontram-se em classe funcional I ou II (NYHA). A avaliação ecocardiográfica, realizada em 16 pacientes, dos 20 em acompanhamento, demonstrou regurgitação aórtica considera- 
KALIL, R. A. K.; TEIXEIRA FILHO, G. F.; SANT'ANNA, J. R. M.; PRATES, P. R.; LUCCHESE, F. A.; BRAUCH, C. R.; PEREIRA, E. M. C.; COSTA, A. R.; SANTOS, M. F.; NESRALLA, I. A. - Recuperação por desbridamento manual da valva aórtica estenótica calcificada. Rev. Bras. Cir. Cardiovasc., $10(2): 65-69,1995$.

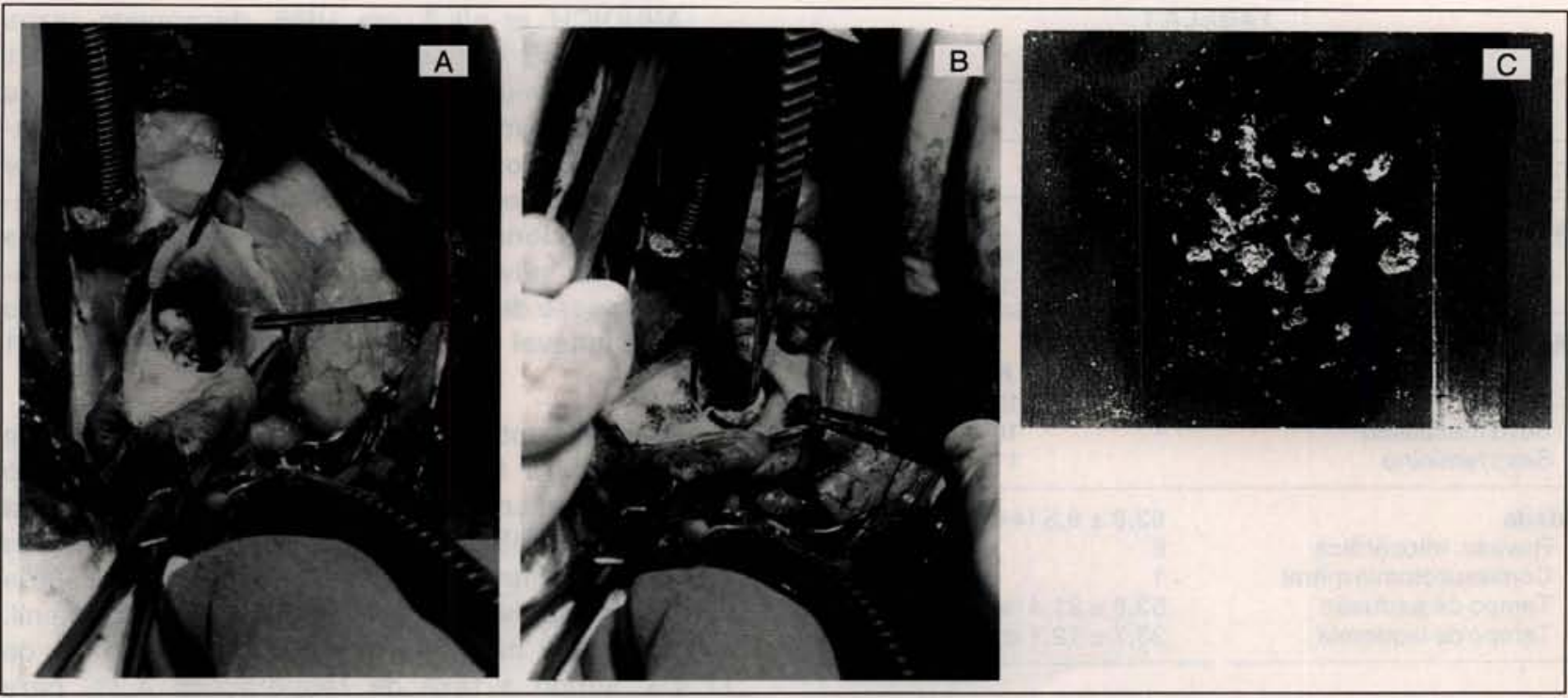

Fig. 2 - Aspectos trans-operatórios: A: valva aórtica bivalvulada severamente calcificada; B: valva aórtica mobilizada após o desbridamento; C:material fragmentado retirado da válvula.

da mínima em 5 casos, leve em 9 e moderada em 2 pacientes. Houve redução do gradiente instantâneo máximo de $90,7 \pm 23,3 \mathrm{mmHg}$ no pré-operatório para $33,0 \pm 7,9 \mathrm{mmHg}$ no pós-operatório $(p<$ $0,01)$. O gradiente médio estimado em $50,3 \pm 11,0$ $\mathrm{mmHg}$ diminuiu para 19,9 $\pm 3,5 \mathrm{mmHg}(\mathrm{p}<0,05)$ (Gráfico 1). A espessura septal medida em 2,0 $\pm 0,5$ $\mathrm{cm}$ no pré-operatório, foi reduzida para $1,2 \pm 0,1$ no pós-operatório ( $p<0,01)$. Ocorreu aumento no volume de ejeção do ventrículo esquerdo de $60,4 \pm$ $9,1 \mathrm{ml}$ para $79,8 \pm 12,5 \mathrm{ml}$ no pós-operatório $(\mathrm{p}<$ 0,01 ) (Gráfico 2). Não ocorreram variações significativas ao ecocardiograma dos diâmetros da aorta e do átrio esquerdo, da espessura da parede posterior do ventrículo esquerdo, da massa ventrícular esquerda e do índice de hipertrofia miocárdica.

\section{COMENTÁRIOS}

O desbridamento mecânico foi utilizado por muito tempo, antes do advento das próteses valvulares artificiais $4,8,11$, tendo sido abandonado praticamente, devido à maior padronização técnica da substituição protética e à incidência de re-estenose a médio prazo 2,5 . O desbridamento ultra-sônico surgido como alternativa técnica ${ }^{10}$, embora com bons resultados imediatos, apresentou como complicação o desenvolvimento de insuficiência aórtica importante em significativa incidência no pós-operatório tardio 3 .

São conhecidas as limitações das próteses disponíveis pois, além dos inconvenientes da anticoagulação nas mecânicas e da durabilidade nas biológicas, quando implantadas em pequenos
GRÁFICO 1

REPRESENTAÇĀO GRÁFICA DA MÉDIA DOS GRADIENTES NA VIA DE SAIDA DO VENTRICULO (INSTANTÂNEO, MÁXIMO E MÉDIO), NAS AVALIAÇŌES PRÉ E PÓS-OPERATÓRIAS

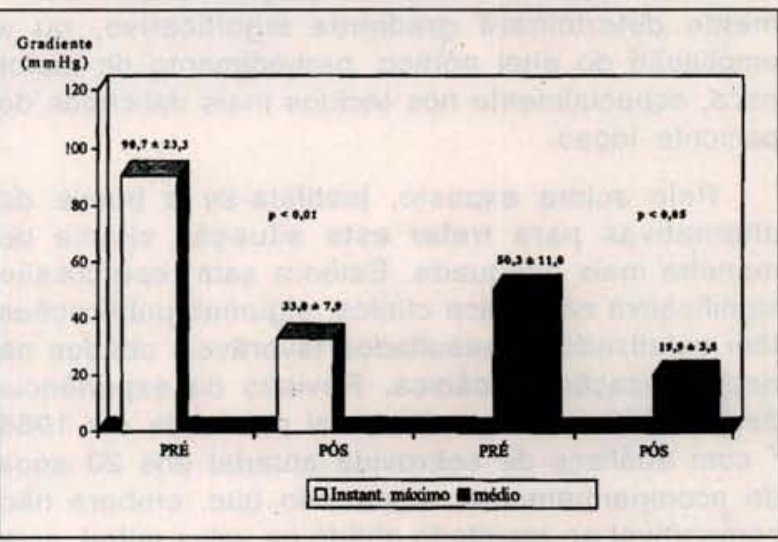

GRÁFICO 2

REPRESENTAÇÃO GRÁFICA DOS VALORES ENCONTRADOS NA AVALIAÇÃO ECOCARDIOGRÁFICA PARA A ESPESSURA SEPTAL VENTRICULAR E O VOLUME DE EJECÃO DO VENTRICULO ESQUERDO, NO PRÉ E PÓS-OPERATÓRIO

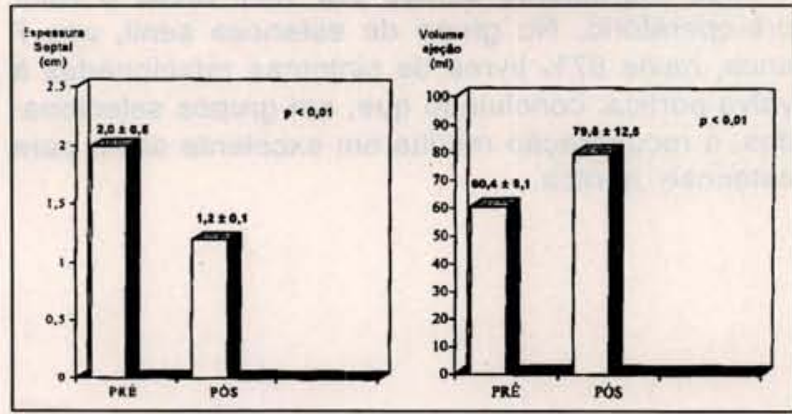


KALIL, R. A. K.; TEIXEIRA FILHO, G. F.; SANT'ANNA, J. R. M.; PRATES, P. R.; LUCCHESE, F. A.; BRAUCH, C. R.; PEREIRA, E. M. C.; COSTA, A. R.; SANTOS, M. F.; NESRALLA, I. A. - Recuperação por desbridamento manual da valva aórtica estenótica calcificada. Rev. Bras. Cir. Cardiovasc., $10(2): 65-69,1995$.

\begin{tabular}{|c|c|c|c|}
\hline \multicolumn{4}{|c|}{$\begin{array}{l}\text { TABELA } 1 \\
\text { CASUISTICA }\end{array}$} \\
\hline & $N^{2}$ & & $\%$ \\
\hline CASOS CONSIDERADOS & 31 & & 100 \\
\hline $\begin{array}{l}\text { Não Recuperados } \\
\text { Congênitos } \\
\text { Senil }\end{array}$ & 10 & $\begin{array}{l}9 \\
1\end{array}$ & 32 \\
\hline $\begin{array}{l}\text { Recuperados } \\
\text { Congênitos } \\
\text { Senil } \\
\text { Sexo masculino } \\
\text { Sexo feminino }\end{array}$ & 21 & $\begin{array}{c}8 \\
13 \\
10 \\
11\end{array}$ & 68 \\
\hline $\begin{array}{l}\text { Idade } \\
\text { Revasc. miocárdica } \\
\text { Comissurotomia mitral } \\
\text { Tempo de perfusão } \\
\text { Tempo de isquemia }\end{array}$ & \multicolumn{3}{|c|}{$\begin{array}{l}63,8 \pm 9,5(44 \mathrm{a} \\
6 \\
1 \\
53,8 \pm 21,4 \mathrm{~min} \\
33,7 \pm 12,1 \mathrm{~min}\end{array}$} \\
\hline
\end{tabular}

orifícios, determinam gradiente pressórico não desprezível e, por vezes, elevado 6 . As estenoses aórticas calcificadas, principalmente as de etiologia senil, apresentam-se com pequenos orifícios para implante de próteses, o que leva o cirurgião a optar entre um substituto valvular pequeno, que certamente determinará gradiente significativo, ou a ampliação do anel aórtico, procedimento de maior risco, especialmente nos tecidos mais delicados do paciente idoso.

Pelo acima exposto, justifica-se a busca de alternativas para tratar esta situação clínica de maneira mais adequada. Embora sem repercussão significativa na prática clínica, algumas publicações têm enfatizado os resultados favoráveis obtidos na descalcificação mecânica. Revisão da experiência desde 1959 na Mayo Clinic foi publicada em 1986 7 com análises de sobrevida atuarial aos 20 anos de acompanhamento, mostrando que, embora não comparável ao resultado obtido na valva mitral, com seleção apropriada, a descalcificação poderia ser uma alternativa aceitável para muitos pacientes. Em 48 pacientes acompanhados por cateterismo cardiaco seriado. SHAPIRA et alii 12 encontraram reestenose tardia como esperada e mais freqüênte nos grupos de doença congênita e reumática; após 5 anos o gradiente aórtico era 1,41 vezes o valor pré-operatório. No grupo de estenose senil, aos 7 anos, havia $87 \%$ livres de sintomas relacionados à valva aórtica, concluindo que, em grupos selecionados, a recuperação resulta em excelente alívio para estenose aórtica.
MINDICH et alii ${ }^{9}$, em 1986, descrevem casos com diâmetros aórticos pequenos (menores de 21 $\mathrm{mm}$ ) ou contra-indicação para anticoagulação, nos quais foi realizada descalcificação com acompanhamento até 6 anos de pós-operatório, concluindo por considerar o procedimento valioso e efetivo em pacientes selecionados. Recomenda-se como fatores de sucesso: valvas trivalvuladas, abandono da tentativa em caso de lesão do tecido, evitar regurgitação residual notável e obter orifício final maior que 1,1 $\mathrm{cm}^{2}$.

Mais recentemente, alguns Serviços têm se motivado para tentar o desbridamento mecânico antes da resseç̧ão primária da valva aórtica calcificada. WEISCHELBAUM et alii ${ }^{13}$, em Buenos Aires, apresentam 67 casos operados entre 1988 e 1993 , consecutivamente, por estenose aórtica senil. A mortalidade hospitalar foi de $5,9 \%$ e a tardia de $17,4 \%$, sendo a taxa de reoperações $4,3 \%$ para pacientes/ano, no acompanhamento de até 57 meses. Dos sobreviventes, $92,3 \%$ estão assintomáticos. Não houve endocardite infecciosa, nem foi usado anticoagulante no pós-operatório. A técnica é decidida no ato cirúrgico, quando as condições anatômicas permitem, fundamentalmente anel aórtico pequeno.

Nossa experiência com a série aqui relatada está em acordo com os resultados publicados pelos autores acima citados. Obtivemos maior sucesso trans-operatório em estenoses senís, embora significativo número de valvas congenitamente estenóticas e severamente calcificadas tenham sido recuperadas. Houve redução do gradiente aórtico a níveis comparáveis à média das próteses, mas certamente inferiores àquelas de pequeno diâmetro, portanto com melhor resultado hemodinâmico. Nenhum paciente necessitou reoperação, no seguimento pós-operatório.

Não tivemos endocardite infecciosa ou outra complicação significativa relacionada à valva. $O$ único óbito foi por intercorrências não cardíacas. O tempo de perfusão requerido para recuperação foi menor que a média, para substituição por prótese. $O$ estudo ecocardiográfico demonstra a significativa e estável melhora hemodinâmica. Nenhum paciente recebeu anticoagulação no pós-operatório. Estes resultados iniciais justificam a manutenção do método como alternativa terapêutica em todos os casos de estenose aórtica calcificada, mas especialmente em idosos com valvas bivalvuladas ou trivalvuladas calcificadas. 
KALIL, R. A. K.; TEIXEIRA FILHO, G. F.; SANT'ANNA, J. R. M.; PRATES, P. R.; LUCCHESE, F. A.; BRAUCH, C. R.; PEREIRA, E. M. C.; COSTA, A. R.; SANTOS, M. F.; NESRALLA, I. A. - Recuperação por desbridamento manual da valva aórtica estenótica calcificada. Rev. Bras. Cir. Cardiovasc., 10 (2): 65-69, 1995.

\section{RBCCV 44205-260}

KALIL, R. A. K.; TEIXEIRA FILHO, G. F.; SANT'ANNA, J. R. M.; PRATES, P. R.; LUCCHESE, F. A.;BRAUCH, C. R.; PEREIRA, E. M. C.; COSTA, A. R.; SANTOS, M. F.; NESRALLA, I. A. - Manual debridement for repair in calcified aortic stenosis. Rev. Bras. Cir. Cardiovasc., 10 (2): 65-69, 1995.

ABSTRACT: A series of 21 patients submitted to mechanical debridement for treatment of calcified stenotic aortic valves is presented. There were 8 congenital and 13 senile valves, in 10 male and 11 female patients. Mean age $63.8 \pm 9.5$ ( 44 to 78 ) years. Six had also coronary bypass grafts and 1 mitral comissurotomy. Total perfusion time was $53.8 \pm 21.4 \mathrm{~min}$ and ischemic time $33.7 \pm 12.1 \mathrm{~min}$. All patients had been followedup from 1 month to 2 years. In 16 a late echocardiographic evaluation was performed. There have been no early and 1 late death. Surviving patients are in functional classes I or II. Maximum instantaneous gradient dropped from $90.7 \pm 23.3 \mathrm{mmHg}$ to $33.0 \pm 7.9 \mathrm{mmHg}(\mathrm{p}<0.01)$ and mean aortic gradient from $50.3 \pm 11.0 \mathrm{mmHg}$ to 19.9 $\pm 3.5 \mathrm{mmHg}(p<0.05)$. Septal width reduced from $2.0 \pm 0.5 \mathrm{~cm}$ to $1.2 \pm 0.1(p<0.01)$. Aortic regurgitation, by echocardiography, was considered minimum in 5 , mild in 9 and moderated in 2 cases. Mechanical debridement of calcified aortic valves, congenital or senile, provides good medium-term relief for aortic stenosis. This technical alternative may be considered in older patients, small aortic roots and higher risks of anticoagulation.

DESCRIPTORS: Aortic stenosis, surgery, debridement. Heart valves, sugery

\section{REFERÊNCIAS BIBLIOGRÁFICAS}

BAEZA, O. R.; MAJED, N. K.; CONROY, D. P.; DONAHOO, J. S. - Combined conventional mechanical and ultrasonic debridement for aortic valvular stenosis. Ann. Thorac. Surg., 54: 62-67, 1992.

COSGROVE, D. M.; RATLIFF, N. B.; SCHAFF, M. V.; EDWARDS, W. D. - Aortic valve decalcification: history repeated with a new result. Ann. Thorac. Surg., 49: 689-690, 1990. (Editorial).

CRAVER, J. M. - Aortic valve debridement by ultrassonic surgical aspirator: a word of caution. Ann. Thorac. Surg., 49: 746-753, 1990.

ENRIGHT, L. P.; HANCOCK, E. W.; SCHUMWAY, N. E. - Aortic debridement: long-term follow-up. Circulation, 44 (Supl. 1): 68-72, 1971.

HILL, D. G. - Long-term results of debridement valvotomy for calcific aortic stenosis. J. Thorac. Cardiovasc. Surg., 65: 708-711, 1973.

6 KALIL, R. A. K.; PRATES, P. R.; LUCCHESE, F. A.; SANT'ANNA, J. R.; SALES, J. B.; ABRAHÄO, R.; NESRALLA, I. A. - Serão despresíveis os gradientes de próteses aórticas? Arq. Bras. Cardiol., 59 (Supl. 2): 73, 1992. (Resumo).
7 KING, R. M.; PLUTH, J. R.; GIULIANI, E. R.; PICHLER, J. M. - Mechanical decalcification of the aortic valve. Ann. Thorac. Surg., 42: 269-272, 1986.

8 KIRKLIN, J. W. \& MANKIN, H. T. - Open operation in the treatment of calcific aortic stenosis. Circulation, 21: $578-586,1960$.

9 MINDICH, B. P.; GUARINO, T.; GOLDMAN, M. E. Aortic valvuloplasty for acquired aortic stenosis. Circulation, 74 (Supl. 1): 130-135, 1986.

10 MINDICH, B. P.; GUARINO, T.; KRENZ, M.; LI, X.; GONZÁLEZ, E. - Aortic valve salvage utilizing high frequency vibratory debridement. JACC, 11: $3 \mathrm{~A}$, 1988. (Resumo).

11 MULDER, D. G. \& WINFIELD, M. E. - Valvuloplasty for acquired aortic stenosis. Ann. Thorac. Surg., 151: 203-215, 1960.

12 SHAPIRA, N.; LEMOLE, G. M.; FERNANDEZ, J.; DAILY, P. O.; DEMBITSKY, W. P.; O'YEK, V.; HAGHIGHI, P.; STEWART, J.; MARSCH, D. G.; BLOOR, C. M. - Aortic valve repair for aortic stenosis in adults. Ann. Thorac. Surg., 50: 110-120, 1990.

13 WEISCHELBAUM, E.; GABE, E.; FIGAL, J. C.; ZAIDMAN, C. L.; CORS, J.; BRUNO, C.; FAVALORO, R. G. - Estenosis valvular aórtica severa calcificada senil: reparación plastica con descalcificación manual. Cardiol. Internat. (Panamá) - (No prelo). 